\title{
A direct repeat of E-box-like elements is required for cell-autonomous circadian rhythm of clock genes
} Yasukazu Nakahata ${ }^{1}$, Mayumi Yoshida ${ }^{1,2}$, Atsuko Takano1, Haruhiko Soma ${ }^{3}$, Takuro Yamamoto ${ }^{3}$, Akio Yasuda ${ }^{3}$, Toru Nakatsu ${ }^{4}$ and Toru Takumi*1,5

\begin{abstract}
Address: ${ }^{1}$ Osaka Bioscience Institute, Suita, Osaka 565-0874, Japan, ${ }^{2}$ Kyoto University Graduate School of Biostudies, Sakyo, Kyoto $606-8501$ Japan, ${ }^{3}$ Life Science Laboratory, Material Laboratories, Sony Corporation, Shinagawa, Tokyo 144-0001, Japan, ${ }^{4}$ Department of Structural Biology, Graduate School of Pharmaceutical Sciences, Kyoto University, Sakyo, Kyoto 606-8501, Japan and ${ }^{5}$ Department of Molecular Neuroscience, Kyoto University Graduate School of Medicine, Sakyo, Kyoto 606-8501, Japan

Email: Yasukazu Nakahata - yasu_nakahata@yahoo.co.jp; Mayumi Yoshida - mayoc591-lj@infoseek.jp; Atsuko Takano - takano@obi.or.jp; Haruhiko Soma - haruhiko.soma@jp.sony.com; Takuro Yamamoto - Takuro.Yamamoto@jp.sony.com; Akio Yasuda - yasuda@sony.de; Toru Nakatsu - nakatsu@pharm.kyoto-u.ac.jp; Toru Takumi* - takumi@obi.or.jp

* Corresponding author
\end{abstract}

Published: 4 January 2008

BMC Molecular Biology 2008, 9:1 doi:10.1 186/147|-2199-9-1
Received: 12 July 2007

Accepted: 4 January 2008

This article is available from: http://www.biomedcentral.com/l47/-2199/9/I

(c) 2008 Nakahata et al; licensee BioMed Central Ltd.

This is an Open Access article distributed under the terms of the Creative Commons Attribution License (http://creativecommons.org/licenses/by/2.0), which permits unrestricted use, distribution, and reproduction in any medium, provided the original work is properly cited.

\begin{abstract}
Background: The circadian expression of the mammalian clock genes is based on transcriptional feedback loops. Two basic helix-loop-helix (bHLH) PAS (for Period-Arnt-Sim) domain-containing transcriptional activators, CLOCK and BMALI, are known to regulate gene expression by interacting with a promoter element termed the E-box (CACGTG). The non-canonical E-boxes or E-box-like sequences have also been reported to be necessary for circadian oscillation.
\end{abstract}

Results: We report a new cis-element required for cell-autonomous circadian transcription of clock genes. This new element consists of a canonical E-box or a non-canonical E-box and an Ebox-like sequence in tandem with the latter with a short interval, 6 base pairs, between them. We demonstrate that both E-box or E-box-like sequences are needed to generate cell-autonomous oscillation. We also verify that the spacing nucleotides with constant length between these $2 \mathrm{E}$ elements are crucial for robust oscillation. Furthermore, by in silico analysis we conclude that several clock and clock-controlled genes possess a direct repeat of the E-box-like elements in their promoter region.

Conclusion: We propose a novel possible mechanism regulated by double E-box-like elements, not to a single E-box, for circadian transcriptional oscillation. The direct repeat of the E-box-like elements identified in this study is the minimal required element for the generation of cellautonomous transcriptional oscillation of clock and clock-controlled genes.

\section{Background}

Circadian rhythms, periodicities with an approximate 24$\mathrm{h}$ length, are essential physiological functions in almost all organisms on the earth and are generated by endogenous biological clocks. The biological clocks consist of 3 components: input, pacemaker, and output. The endogenous period length is not exactly $24 \mathrm{~h}$ and must be entrained by light. In mammals, other entrainment cues such as food intake or temperature, even social cues, can reset the circadian rhythm. The central oscillator in mam- 
mals is located in the suprachiasmatic nuclei (SCN) in the anteroventral hypothalamus. Recent studies revealed that the peripheral clocks reside not only in other brain regions but also in peripheral organs and even in cultured cells [1-4]. The output of the biological clocks includes broad physiological phenomena such as locomotor activity, sleep-wake cycle, hormonal secretion, cardiovascular condition, bowel movement, and even mental states. The disturbance of the biological clocks may cause many dysfunctions from routinely experienced jet-lag or work-shift problems to sleep disturbance and cardiovascular, metabolic or mental diseases $[2,5]$.

Recent molecular biological studies have revealed that the molecular mechanism of the biological clocks is based on the interlocked loops of transcriptional and translational feedback [6-8]. Among the transcriptional controls in mammals, 3 major clock components for canonical clock genes, i.e., E-box, RORE, and DBPE, have been reported so far $[3,9]$. The E-box (CACGTG), for example, in the Per1 promoter, is the most well-known regulatory element. It is considered the binding site for the heterocomplex of CLOCK/BMAL1, which is a positive regulatory element. Negative regulatory elements, PERs and CRYs, restrain the transcriptional activity [10-12]. It is widely known that not only other clock genes are regulated by the E-box but also output regulators or clock-controlled genes are regulated by it as well [13-17]. On the other hand, Bmal1, whose circadian expression exhibits a sort of anti-phase to Per2 expression, is positively regulated by Ror $\alpha$ and negatively regulated by Rev-erb $\alpha$, through RORE [18-21].

Per2 was literally cloned as a second mammalian period gene $[22,23]$, however, gene-knockout analysis revealed a more prominent role for mPER2 in the mammalian clock than PER1 [24]. Using the in vitro rhythm oscillation monitoring system (IV-ROMS) [3,25], instead of the canonical E-box (CACGTG), we previously identified an E-box-like sequence (CACGTT) in the Per2 promoter region in vitro [26], which is essential for the cell-autonomous transcriptional oscillation of Per2 as well as for Per2 circadian oscillations in vivo [27]. For the cell-autonomous transcriptional oscillation of Per2, not only this Ebox-like sequence but also the downstream region is involved in the circadian oscillation of Per2 [26]. Here, we identified closely spaced E-box or E-box-like elements in the regulatory regions of canonical clock genes including Per2. Our comprehensive approach raised the possibility that not just a single E-box but a direct repeat of E-box-like elements is required for circadian oscillation of core clock and clock-controlled genes.

\section{Results}

Our previous study demonstrated that an E-box-like sequence (CACGTT) and its downstream region are essen- tial for transcriptional oscillation of Per2, a crucial component of molecular clocks [26]. Comparing with other mammalian genome sequences, within the conserved regions among mammalian (human, rhesus, rat, mouse, and opossum) genomes, we found that an E-box-like sequence followed by another non-canonical E-box with 6-base pairs (bp) between the two (Fig. 1A). In addition to Per2, we found a similar E-box or E-box-like sequence followed by another E-box-like sequence with an intervening space of $6 \mathrm{bp}$ in Per1 and Per3, which are other mammalian period homologues, in their upstream promoter region close to the transcription start site (TSS). The E-box or E-box-like sequences distal and proximal to TSS were dubbed E1 and E2, respectively, and collectively as the "EE-element" (Fig. 1A). These sequences are well conserved among diverse mammals, such as human, rhesus, rat, and mouse, and even the opossum which is evolutionally located at the farthest branch from humans among the mammals. To examine whether these EE-elements are involved in transcriptional oscillation, we performed the IV-ROMS established in our previous study by using the following constructs: The oligonucleotides of 3 (hPer1, $h P e r 2$, and $h P e r 3$ ) EE-elements were inserted upstream of the SV40 promoter in a luciferase (luc) plasmid, and the constructed plasmids were used to transfect mouse NIH3T3 cells. All 3 EE-elements gave significant oscillation of luciferase activity but the amplitudes were different among the genes (Fig. 1B). The hPer2 EE-element displayed the most robust oscillation, whereas the hPer1 EE-element gave the lowest amplitude, which correlated with the patterns derived from $l u c$-constructs containing the longer promoter regions [26]. The amplitude in circadian oscillation seemed to correlate with the number of mismatches in the E2 sequence. In fact, a mutant hPer3 EE-element, in which the GAG at the end of the E2 sequence was replaced with canonical GTG, exhibited a higher amplitude than the wild-type hPer3 EE-element (see Additional file 1).

To investigate whether both E1 and E2 were necessary for generating the cell-autonomous oscillation, we again performed IV-ROMS with the use of the mutant $h$ Per2 EE-element constructs. Not only the double E-box-like mutations (E1mE2m) but also the single E-box-like mutation $(\mathrm{E} 1 \mathrm{~m}$ or $\mathrm{E} 2 \mathrm{~m})$ resulted in drastically reduced circadian transcription or led to an almost complete loss of transactivation (Fig. 2). A longer Per2 promoter ( $2 \mathrm{~kb}$ ) with double E-element mutants showed that the amplitude was heavily reduced, with circadian transcription hardly being observed (see Additional file 2). These results are similar to those obtained with mutations of 2 ROREs in the Bmal1 promoter [21] and suggest that these 2 E-box-like elements function in Per2 transactivation. 


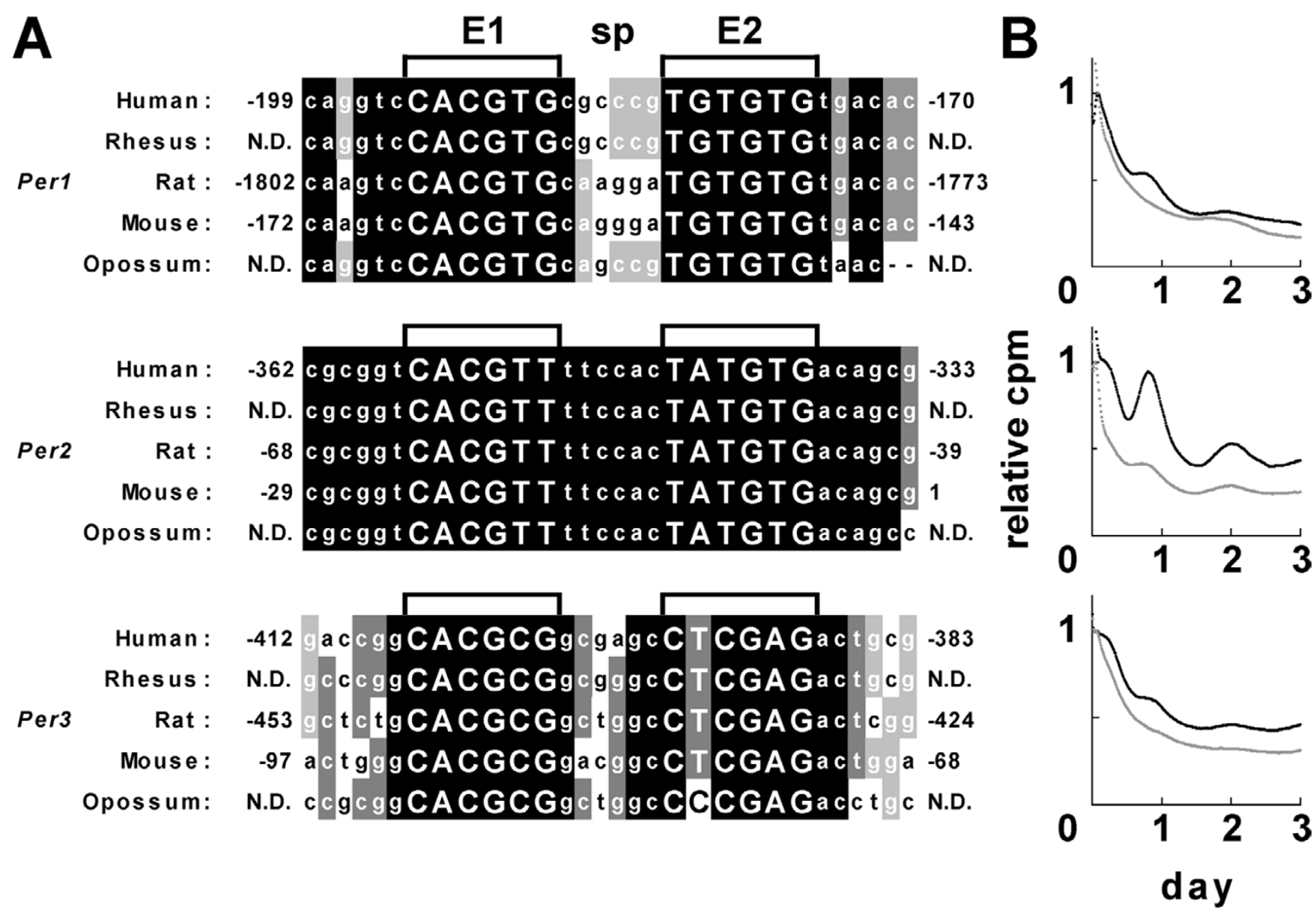

Figure I

The "EE-element" possesses transcriptional activity displaying a circadian rhythm. (A) Sequence alignments of the promoter region, containing each EE-element of Perl, Per2 and Per3 gene, among human, rhesus, rat, mouse and opossum (The $\mathrm{NCBI}$ accession numbers are indicated in Additional file 6). The core sequences (EI and E2) within the EE-element are shown in capital letters. Numbers at both sides of alignments indicate the position from the transcription start site (TSS). (B) EE-element-driven luciferase bioluminescence by IV-ROMS. Black lines indicate bioluminescence after serum shock; and gray lines show the negative control (replacement with same medium). Abscissa presents "day"; and ordinate, "relative luciferase intensity". First peak values of the curves were set to $\mathrm{I}$.

To see whether endogenous clock transcription factors, BMAL1 and CLOCK, could bind to the EE-element, we investigated the binding of endogenous BMAL1 and CLOCK to hPer2 double-stranded DNA fragment immobilized on streptoavidin beads (Fig. 3). For this experiment, we raised specific antibodies against BMAL1 (see Additional file 3). Mouse liver lysates were prepared at 4$\mathrm{h}$ intervals and immunoblotted with antibodies against BMAL1 or CLOCK (indicated as PC in Fig. 3A). When the lysates were incubated with the bead-bound Per2 oligonucleotides and electrophoresis performed, followed by immunoblotting, bands corresponding to BMAL1 and CLOCK were detected (Fig. 3A). The shifted bands seen correspond to phosphorylated BMAL1; and its phosphorylation level peaked around at CT (circadian time) 12, which is consistent with our previous data obtained with the Per2 promoter [26]. In contrast, the oligonucleotides with the same length but having scrambled sequences bound neither BMAL1 nor CLOCK proteins. The amounts of BMAL1 and CLOCK pulled down with mutant E1m or with the double-mutant E1mE2m were drastically reduced or almost undetectable compared with those pulled-down by the wild-type EE-element (Fig. 3B). This result suggests that the endogenous BMAL1 and CLOCK proteins bound to the E1 element but not to E2. On the other hand, when we used the E2m oligonucleotide, precipitated BMAL1 and CLOCK proteins were substantially detected. These results suggest that the binding of CLOCK/BMAL1 to E1 and that of unknown factors to E2 are necessary for cell-autonomous circadian transcription. 
A

E1

E2

\section{hPer2wt 5'-cgcgg t CACGTT t t ccac TATGTGacagcg-3'

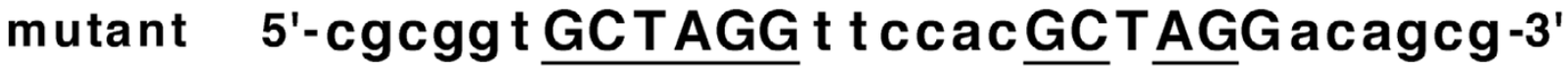
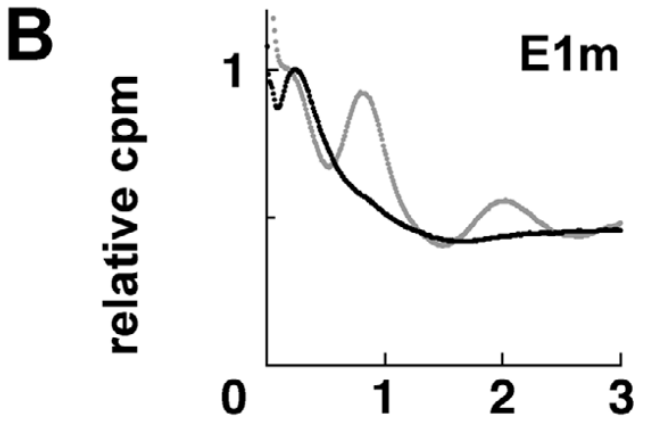

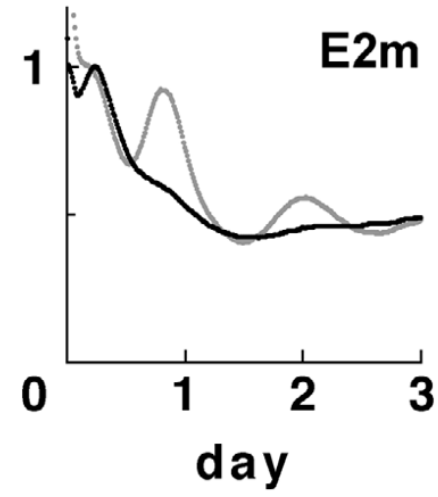

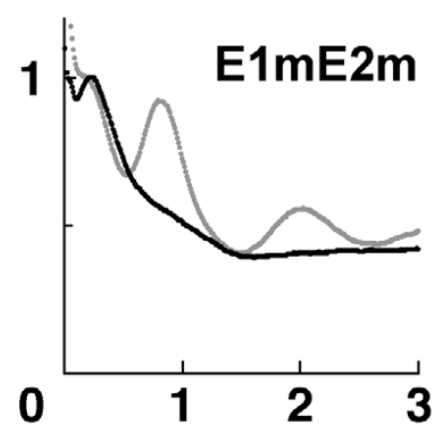

Figure 2

Effects of EE-element mutants on circadian expression of luciferase. (A) Wild-type (upper, gray letters) and mutant (bottom, black letters) sequences of hPer2 EI and E2 and their flanking sequences are shown. Underlines indicate sequences changed from the wild-type one. The core sequence within the EE-element is shown in capital letters. (B) EE-element-driven luciferase bioluminescence by IV-ROMS. Gray lines indicate bioluminescence of wild-type hPer2 EE-element; and black lines, those of mutants. The abscissa presents "day", and the ordinate indicates "relative luciferase intensity". First peak values of the curves were set to $I$.

We next examined the length of the space between E1 and $\mathrm{E} 2$, because the spaces between two E-box-like elements were 6 bp in all of 3 Pers EE-elements (Fig. 1A). To see whether the space length was crucial for cell-autonomous circadian transcription, we constructed different spaces (4 8 bp) between E1 and E2 of the hPer2 EE-element and performed IV-ROMS (Fig. 4). sp7, in which there were 7 bp spacing nucleotides between the 2 E-elements, yielded a circadian rhythmicity of Per 2 transcription but with a slightly diminished amplitude compared with the wildtype oligonucleotide. The amplitude of sp5 and sp8 (5-bp and 8-bp space, respectively) or sp4 (4-bp space) displayed almost no or no oscillation of Per2, respectively. These results demonstrate that the spacing between the 2 E-elements was critical for rhythm generation and that the 6-bp (or 7-bp) space was necessary for cell-autonomous transcriptional oscillation. Thus the EE-element for circadian rhythm may be defined as follows: 1) E1 and E2 allow 1 and 2 base mismatches, respectively, and 2) the space between the 2 E-elements is 6 or 7 bp (Table 1 ).

Previous studies [28-30] in which clock or clock-controlled genes were extensively examined at the transcriptional level by DNA microarrays revealed that a total of 1551 genes exhibited circadian oscillation. So we examined how many genes contained the EE-element that met our criteria described above in their promoter regions within
$1 \mathrm{~kb}$ upstream from the TSS. As a consequence of in silico analysis, 30 EE-elements in 29 genes were found among 1510 genes, which sequences are available from the VISTA database (Table 2). The genes that appeared in different papers and organs were clock and clock-controlled genes, such as Nr1d1 (also known as Rev-erb $\alpha$ ), Dbp, Avp and Tef, besides Per2. This result strongly suggests that not a single E-box but a direct repeat of E-box-like elements is essential for the generation of cell-autonomous circadian oscillation. To confirm this, by using IV-ROMS we investigated whether the EE-element in $D b p$ was also required for its rhythmicity. The hDbp EE-element showed circadian oscillation as well as the hPer2 one (see Additional file 4). The mutants of E1 and/or E2 in the EE-element as well as the space mutants, sp4, sp5, and sp8 displayed no rhythmicity (see Additional file 4), which is consistent with the results of Per2 described above.

\section{Discussion}

Among the genes identified by in silico analysis (Table 2), arginine vasopressin $(A v p)$ is a neuropeptide physiologically synthesized in the hypothalamus in a circadian manner but pathologically expressed by small-cell lung cancer. In the latter case, upstream stimulatory factor (USF), another bHLH transcription factor, activates the vasopressin promoter via the adjacent non-canonical E-boxes in tandem with a 6-nucleotides space between them and 


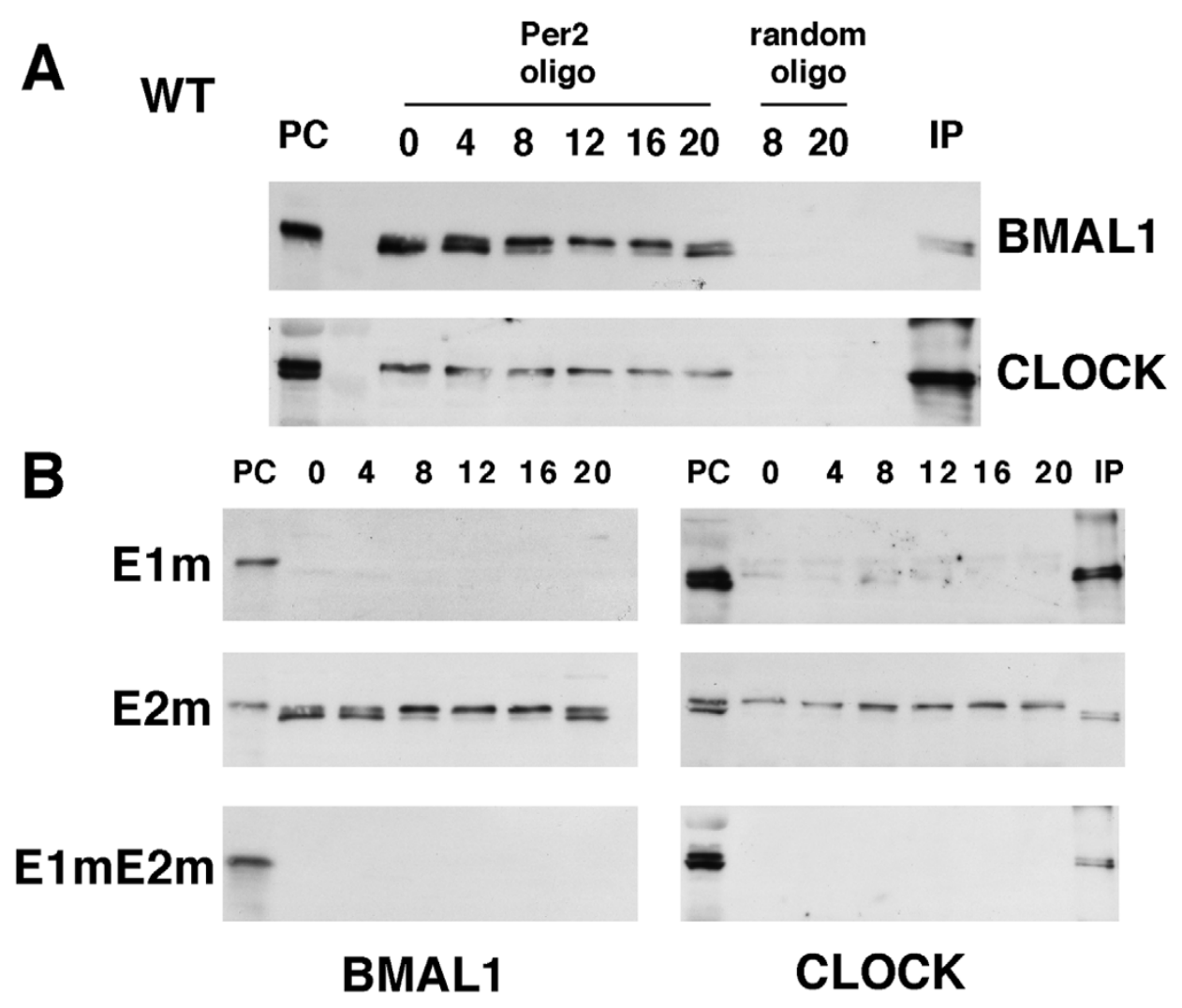

\section{Figure 3}

Pull-down assays by using oligonucleotides. (A) Both BMALI and CLOCK proteins bind to Per2 oligonucleotide. BMALI is phospholrylated in a circadian manner, whereas no binding to the random oligonucleotide occurs. Mouse liver lysates at $C T$ 16 were electrophoresed and immunoblotted with anti-BMALI and anti-CLOCK (PC) and immunoprecipitated by antiCLOCK and blotted by either BMALI or CLOCK (IP). WT; wild-type $h$ Per2 oligonucleotide, EI; EI mutant oligonucleotide, E2; E2 mutant oligonucleotide, EImE2m; EI, E2 double mutant oligonucleotide. (B) As for BMALI, the binding to E2m is clearly observed; whereas no binding to either EIm or EImE2m is detected. For CLOCK, the binding to E2m is observed; however, the binding to EIm is hardly seen and no binding to $E I m E 2 m$ is evident.

the second E-box is important for full enhancer function of the first E-box [31]. Furthermore, hypoxia-inducible factor 1 (HIF-1), a bHLH-PAS transcription factor, has recently been reported to regulate some hypoxia-inducible genes such as vascular endothelial growth factor (VEGF) through an HIF-1 binding site and its downstream HIF-1 ancillary sequence, both of which are non-canonical E-boxes [32]. The space between these motifs in this case is 8 nucleotides and is crucial for activity of promoter.
Not only mammalian genes but also fly clock genes contain a direct repeat of E-box-like elements, as discovered in this study. The Drosophila timeless promoter includes an Ebox-like sequence dubbed TER1 and is considered as an EE-element together with its 7-bp downstream canonical E-box. This TER1 plays a major role in tim transcription [33]. These reports in addition to this study demonstrate that not a single E-box but an EE-element is crucial for

Table I: The criteria for the EE-element. Criteria for the EE-element are as follow: I) 2 E-boxes, close to each other, are needed, 2) EI E-box allows I base mismatch, E2 E-box allows 2 base mismatches, 3) the spacing between 2 E-boxes is 6 or 7 bp, and 4 ) the element is located within I kb upstream region from the TSS.

\begin{tabular}{ccc}
\hline E-box & space & E-box \\
\hline CACG T G & 6 or 7 bp & CACG T G \\
$0 \sim 1$ mismatch & & $0 \sim 2$ mismatch \\
\hline
\end{tabular}




\begin{tabular}{|c|c|c|c|}
\hline$A$ & E1 & sp & E2 \\
\hline sp4 & 5'-cgcggt CACGTT & ttac & TATGTGacagcg-3' \\
\hline sp5 & 5'-cgcggt CACGT T & ttcac & 'TATGTGacagcg-3' \\
\hline Per2 wt & $5^{\prime}-\operatorname{cgcgg} t \mathrm{CACG} T \mathrm{~T}$ & ttccac & 'TATGTGacagcg-3' \\
\hline & 5'-cgcggt CACGTT & ttctcac & 'TATGTGacagcg-3' \\
\hline sp8 & CACGTT & tectcac & TATGTGacagcg-3' \\
\hline
\end{tabular}

B
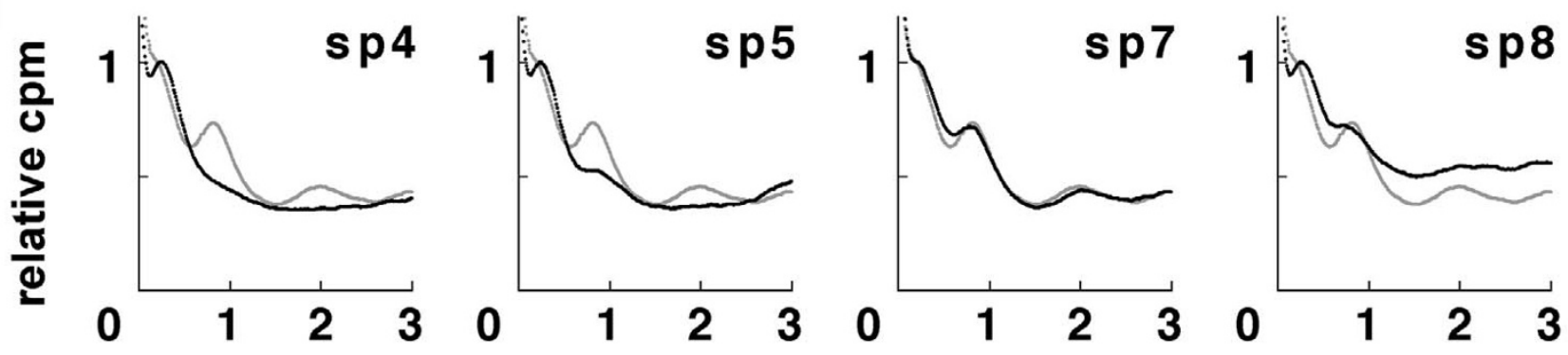

day

Figure 4

The space between the 2 E-box-like sequences is critical for cell-autonomous circadian transcription. (A) Wildtype (sp6; gray letters) and mutant (sp4, 5, 7, 8; solid letters) sequences of the hPer2 EE-element and their flanking sequences are shown. Underlines indicate nucleotide(s) inserted into the space of the EE-element. The core sequence within the EE-element is shown in capital letters. (B) EE-element-driven luciferase bioluminescence detected by IV-ROMS. Black lines indicate bioluminescence of mutant hPer2 EE-elements; and gray lines, those of the wild type. The abscissa presents "day"; the ordinate shows "relative luciferase intensity". First peak values of the curves were set to I.

transcriptional activation by certain bHLH transcription factors.

Our results suggest that 2 dimeric complexes of transcription factors bind to the 2 E-box-like elements with a short distance between them, such as 6 nucleotides. To support this possibility, we simulated this binding of bHLH transcription factors to the EE-element on the DNA by using the crystal structure of the bHLH domain of the Myc-Max heterodimer bound to DNA (PDB ID: 1NKP) [34]. Since 1 turn of double-helix DNA is approximately $34 \AA$ and requires 10.4 nucleotides, the distance from the beginning of E1 to the end of space, which consists of 12 nucleotides, spans $39.2 \AA(34 / 10.4 \times 12$, Fig. 5A). Two binding faces form a 55.8-degree angle (360/10.4 × 12-360, Fig. $5 \mathrm{~B})$. The maximum dimension of the bHLH structure, which lies along the DNA, is about $32 \AA$ (Fig. 5C). This dimension is within the $39 \AA$ calculated above and allows the binding. When the space is 5 nucleotides, for example, the distance between 2 complexes shortens approximately 3 A horizontally compared with the case of 6 nucleotides; however, the angle between them becomes $20.6^{\circ}$. This precludes the possibility that the bHLH structure binds to the EE-element, because 2 whole structures including PAS domains may collide with each other. This simulation supports our contention that 6 nucleotides between 2 Ebox-like elements, not 5 nucleotides, allow the binding of bHLH transcription factors to the EE-element on the DNA.

As a candidate for unknown factors that bind to E2, another type of transcription factor can be a possible candidate as well as the bHLH family transcription factor including HIF1 [35] or the bZip family transcription factor E4BP4 (also known as NFIL3) [36]. Our preliminary EMSA suggests that some proteins may bind to E2 (see Additional file 5). Alternatively, different forms of the CLOCK and BMAL1 complex can be involved in this circadian transcription. The circadian transcriptional complex includes at least CLOCK, NPAS2, BMAL1, PERs and CRYs, probably in different combinations, and these proteins can be posttranslationally modified [37]. While E1 
Table 2: The EE-elements in clock and clock-controlled genes. Clock and clock-controlled genes containing putative EE-elements (A; [28], B; [29], C; [30]). "Phase" means peak time. Phase 2, the peak value = CT 0.1 4.0; phase 6, CT 4.1 8.0; phase 10, CT 8.1 12; phase 14, CT I2.I 16; phase 18, CT 16.I 20; phase 22, CT 20.1 24. "pos" indicates the position of the EE-element from the TSS. Underlines indicate nucleotides matched to the canonical E-box.

\begin{tabular}{|c|c|c|c|c|c|c|c|c|}
\hline \multirow[t]{2}{*}{ Paper } & \multirow[t]{2}{*}{ Organ } & \multirow[t]{2}{*}{ Phase } & \multirow[t]{2}{*}{ Gene } & \multirow[t]{2}{*}{ pos } & \multicolumn{2}{|l|}{ mouse } & \multicolumn{2}{|l|}{ human } \\
\hline & & & & & sequence & sp & sequence & sp \\
\hline$A$ & $\mathrm{SCN}$ & 10 & Per2 & -23 & CACGTTTTCCACTATGTG & 6 & CACGTTTTCCACTATGTG & 6 \\
\hline A & Liver & 14 & & -23 & & & & \\
\hline B & Liver & 14 & & -23 & & & & \\
\hline $\mathrm{B}$ & Heart & 10 & & -23 & & & & \\
\hline $\mathrm{C}$ & SCN & 10 & & -23 & & & & \\
\hline C & Liver & 14 & & -23 & & & & \\
\hline$A$ & $\mathrm{SCN}$ & 18 & Nrldl & -878 & CACGTGAAGCTCTCACGTT & 7 & CACGTGAAGCCCTCACGTT & 7 \\
\hline$A$ & Liver & 6 & & -878 & & & & \\
\hline B & Liver & 6 & & -878 & & & & \\
\hline B & Heart & 6 & & -878 & & & & \\
\hline $\mathrm{C}$ & Liver & 6 & & -878 & & & & \\
\hline B & Liver & 6 & Dbp & -606 & $\underline{\text { CACGCGCAAAGCCATGTG }}$ & 6 & CACGAGCAGAGCCATGTG & 6 \\
\hline B & Heart & 10 & & -606 & & & & \\
\hline C & $\mathrm{SCN}$ & 6 & & -606 & & & & \\
\hline $\mathrm{B}$ & Liver & 10 & Tef & -529 & CACGTGCAGAGCㅡㄷGTG & 6 & CACGTGCGGCGCㅡTCGTG & 6 \\
\hline B & Liver & 10 & & -208 & CACCTGGCCCAGCACGTG & 6 & CACCIGGCCCCGCACGTG & 6 \\
\hline B & Heart & 10 & & -529 & & & & \\
\hline $\mathrm{B}$ & Heart & 10 & & -208 & & & & \\
\hline$A$ & Liver & 10 & Foxo3a & -97 & CACGCGCACTCCCACACG & 6 & $\underline{\text { CACGCGCACTCACACACG }}$ & 6 \\
\hline B & Liver & 10 & & -97 & & & & \\
\hline C & Liver & 10 & & -97 & & & & \\
\hline A & Liver & 10 & 37324I3IIIRik & -307 & CACGTGGTCCCAGCAGGTG & 7 & CACGTGGTCCCTGCAGGTG & 7 \\
\hline C & Liver & 10 & & -307 & & & & \\
\hline A & Liver & 18 & Tfdpl & -916 & CACGTCACCGCGCㅌGCGCG & & CACGTCACCGCGCCGGCGCG & 7 \\
\hline B & Liver & 14 & & -916 & & & & \\
\hline $\mathrm{B}$ & Liver & 18 & Tfrc & -127 & TACGTGCGGAAGGAAGTG & 6 & TACGTGCCTCAGGAAGTG & 6 \\
\hline B & Heart & 2 & & -127 & & & & \\
\hline B & Liver & 22 & Sox3 & -200 & CAGGTGAGAGAAGCCCGCG & 7 & CAGGTGCGAGAAGCCCGCG & 7 \\
\hline B & Heart & 18 & & -200 & & & & \\
\hline $\mathrm{B}$ & Heart & 2 & MGI:|35|465 & -387 & CACGGGCCCCACCETTGTG & 6 & CACGGGCCCCACCTTTGTG & 6 \\
\hline $\mathrm{C}$ & $\mathrm{SCN}$ & 6 & Avp & -186 & CACGTGTGTCCCCAGATG & 6 & CACGTGTGTCCCCAGATG & 6 \\
\hline$A$ & SCN & 14 & Serpinel & -179 & $\overline{\text { CACGTGTCCCAGCAAGTC }}$ & 6 & 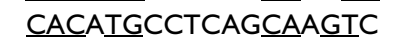 & 6 \\
\hline B & Liver & 6 & Wwp2 & -33 & 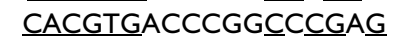 & 6 & 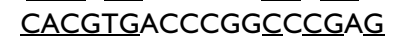 & 6 \\
\hline $\mathrm{C}$ & $\mathrm{SCN}$ & 18 & Hnrpm & -42 & CACGTGAGCGCGCAGGCG & 6 & CACGTGGGCGCGCAGGCG & 6 \\
\hline $\mathrm{B}$ & Heart & 6 & Sn $\times 2$ & -41 & CACGTGACAGGTCCGCGAG & 7 & CACGTGACGGGTCCGCGAG & 7 \\
\hline$A$ & $\mathrm{SCN}$ & 18 & Spag7 & -238 & CACGAGCACTTTCTACTTG & 7 & CACGGGCACTTTCTACTIG & 7 \\
\hline$A$ & $\mathrm{SCN}$ & 10 & Pabpnl & -438 & CACCTGTCGCACAACGGG & 6 & CACCTGTCACGAAACGAG & 6 \\
\hline $\mathrm{B}$ & Heart & 18 & Zic2 & -415 & CAGGTGGAGCGGCGGGTG & 6 & CAGGTGGAGCCGCTGGTG & 6 \\
\hline$A$ & $\mathrm{SCN}$ & 18 & Cacna2d3 & -37 & 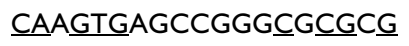 & 7 & 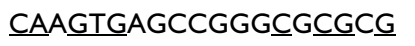 & 7 \\
\hline$A$ & $\mathrm{SCN}$ & 10 & Cog8 & -705 & 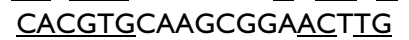 & 7 & 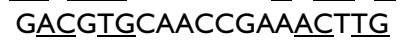 & 7 \\
\hline $\mathrm{B}$ & Heart & 2 & Col4a2 & -641 & CACCTGGCCGTGCCACCCG & 7 & CACCTGGCCGTGCCACGCG & 7 \\
\hline $\mathrm{C}$ & $\mathrm{SCN}$ & 14 & Ube2s & -117 & $\overline{\mathrm{AACGTGCGCGTTGACGTA}}$ & 6 & $\overline{\mathrm{AACGTGCGCGCTGACGTC}}$ & 6 \\
\hline$A$ & SCN & 10 & $\operatorname{lgsf4a}$ & -584 & GACGTGCAAAGCACGCATG & 7 & GACGTGCAAAGCACGCATG & 7 \\
\hline C & $\mathrm{SCN}$ & 18 & Plekhal & -31 & 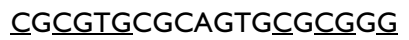 & 7 & 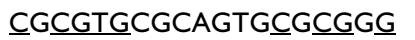 & 7 \\
\hline $\mathrm{B}$ & Heart & 2 & Qk & -352 & CCCGTGGGTGCGCACGCG & 6 & CCCGTGGGTGCGCACGCG & 6 \\
\hline $\mathrm{C}$ & $\mathrm{SCN}$ & 6 & $\operatorname{Rexo2}$ & -27 & $\overline{\mathrm{C}} \mathrm{C} \overline{\mathrm{CGTG}} \mathrm{GGTTTGCGACGTT}$ & 7 & $\overline{\mathrm{C}} \mathrm{C} \overline{\mathrm{CGTG}} \mathrm{GGTTGCGACGTT}$ & 7 \\
\hline $\mathrm{B}$ & Liver & 10 & Trfp & -683 & CGCGTGGCGCGCTCTCGCG & 7 & CGCGTGGTGCGTTCCCGCG & 7 \\
\hline C & Liver & 18 & $\mathrm{BC} 014685$ & -186 & CCCGTGACATTGCCGCGGG & 7 & CCCGTGACGCGGGCGCGGG & 7 \\
\hline $\mathrm{B}$ & Heart & 2 & Ptma & -23 & CGCGTGAGTCCCCCACTGG & 7 & CGCGTGAGTCCCCCACTGG & 7 \\
\hline
\end{tabular}

element is sufficient for binding of CLOCK and BMAL1, it is possible that binding to E2 can occur only after CLOCK and/or BMAL1 originally binds to E1. Thus E2 can be nec- essary for the binding of different forms of the complex or perhaps the binding to E2 is important for complex activation. Further studies, including identification of 


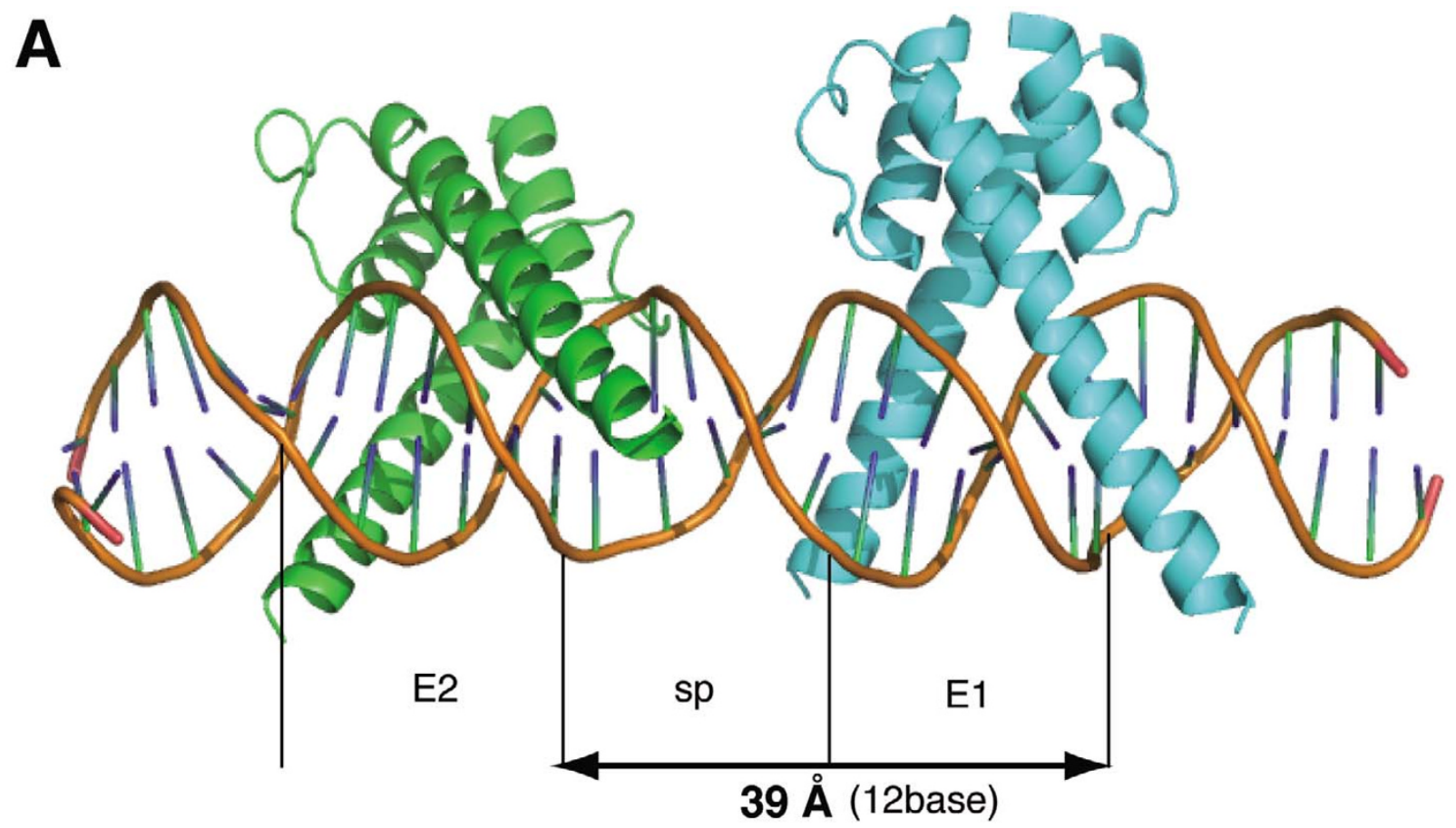

B
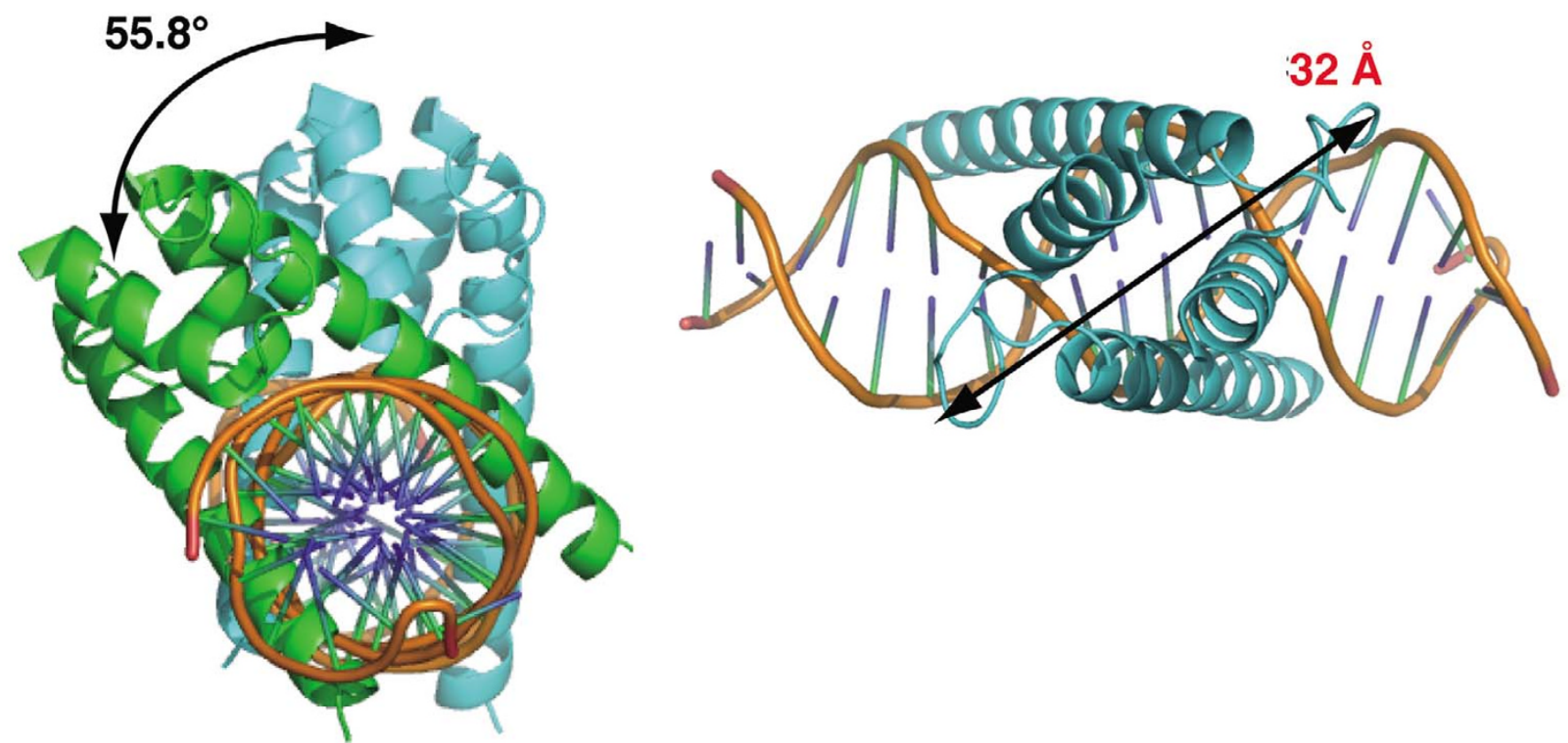

Figure 5

Structural model for the binding of bHLH transcription factors to DNA. (A) Since I turn of double helix DNA is approximately $34 \AA$ and requires 10.4 nucleotides, I EE-element, which consists of 12 nucleotides, spans $39.2 \AA$ ( $34 / 10.4 \times 12$ ). (B) Two binding faces form a 55.8-degree angle (360/10.4 × 12-360). (C) The maximum dimension of the bHLH structure, which lies along the DNA, is about $32 \AA$, which is within the $39 \AA$ calculated above and allows the binding. Green indicates a hetero complex of transcription factors such as BMALI and CLOCK; and light blue, unknown factors. 
unknown factors or the circadian transcription complex and their crystal structures, will be required to elucidate the precise mechanism of transcriptional activation via an EE-element.

\section{Conclusion}

Here we identified a direct repeat of E-box-like elements consisting of a canonical E-box or non-canonical E-box in tandem with an E-box-like element, having a short interval of 6 base pairs between them. Although other regions outside the EE-element seem to be active in robust circadian oscillation with high amplitude [38], the EE-element identified in this study, not a single E-box, is the minimal required element for the generation of cell-autonomous transcriptional oscillation of clock and clock-controlled genes.

\section{Methods \\ Cell culture}

NIT3T3 cells were grown at $37^{\circ} \mathrm{C}$ and $5 \% \mathrm{CO}_{2}$ in Dulbecco's modified Eagle's medium $(1.0 \mathrm{~g} / \mathrm{L}$ glucose $)$ with $\mathrm{L}$ Gln and sodium pyruvate (DMEM, Nacalai tesque, Kyoto, Japan) supplemented with $10 \%$ fetal bovine serum (FBS; ICN Biomedicals, Inc.) and antibiotics.

\section{Antibody}

Rabbit BMAL1 polyclonal antibody was raised against glutathione S-transferase (GST) fused to the amino terminus of BMAL1 (amino acids 1-99). The antiserum was purified through GST and GST-BMAL1 columns. Also, BMAL1 and E4BP4 antibodies were kindly donated by Drs. Teruya Tamaru [39] and Toshiya Inaba [40], respectively. CLOCK antibody (S-19; sc-6927) was purchased from Santa Cruz Biotechnology, Inc.

\section{Construction of the EE-element::SV40 promoter-luciferase plasmid}

The oligonucleotide sequences used in this study are indicated in Additional file 6. Each fragment of the doublestranded oligonucleotides of the hPer genes was cloned into a modified pGL3 (R2.2) promoter vector digested with SacI-NheI. To construct the modified pGL3 (R2.2) promoter vector plasmid, we excised the SV40 promoter fragment from the pGL3 promoter vector (Promega) by digestion with BglII-NcoI and ligated it into the compatible site of pGL3 (R2.2) basic vector (Promega). To rule out the possibility that 2 E-box-like elements in multiple cloning sites of this vector possessed a binding potential for CLOCK/BMAL1, these sequences were removed.

IV-ROMS (in vitro real-time oscillation monitoring system) NIH3T3 cells at $1.0 \times 10^{5}$ plated in Opti-MEM (GIBCO) supplemented with $10 \%$ FBS in $35-\mathrm{mm}$ dishes were transfected with the desired plasmids by using LipofectAMINE and plus reagent (Invitrogen). Twenty-four hours after transfection, the medium was exchanged for serum-rich medium (DMEM supplemented with 50\% newborn calf serum [NBS; JRH]), and $2 \mathrm{~h}$ later this medium was replaced with Opti-MEM supplemented with $1 \%$ FBS and $0.1 \mathrm{mM}$ luciferin/10 mM HEPES ( $\mathrm{pH} 7.2$ ). Bioluminescence was measured by using IV-ROMS as described previously [25].

\section{Oligonucleotide pull-down experiment}

The oligonucleotide pull-down experiment was performed as described previously $[21,26]$. Mouse liver lysate was prepared at 4-h intervals by homogenizing liver in icecold PBS with $2 \mathrm{mM}$ EDTA and $10 \mu \mathrm{g} / \mathrm{ml}$ aprotinin/leupeptin/pepstatin, and $1 \mathrm{mM}$ PMSF as proteinase inhibitors (PIs). The pellet was sequentially incubated in hypotonic buffer (50 mM Tris-HCl pH 7.4, $2 \mathrm{mM} \mathrm{MgCl}_{2}$, $1 \mathrm{mM}$ EDTA, $1 \mathrm{mM}$ DTT, and PIs) and incubation buffer (50 mM Tris-HCl pH 7.4, 50 mM NaCl, 2 mM EDTA, 10\% glycerol, $1 \%$ NP40, $1 \mathrm{mM}$ DTT, $100 \mathrm{mM} \mathrm{NaF}, 2 \mathrm{mM}$ $\mathrm{Na}_{3} \mathrm{VO}_{4}, 10 \mathrm{mM} \mathrm{Na}_{4} \mathrm{P}_{2} \mathrm{O}_{7}$, and PIs). After centrifugation, the supernatant was transferred to a new tube and the remaining pellet was sonicated; and following centrifugation its supernatant combined with the original supernatant as the nuclear extract. The nuclear extracts were incubated with a biotinylated oligonucleotide (see Additional file 6) that had been immobilized on streptavidinSepharose beads (Amersham) for $1 \mathrm{~h}$ at $4^{\circ} \mathrm{C}$. After having been washed with incubation buffer, the resultant complex was subjected to immunoblot analysis. The surgical and experimental procedures performed on mice were approved by the OBI Animal Research Committee.

\section{In silico analysis of the EE-element}

The gene list for this analysis was derived from the reports of microarray analyses [28-30]. The genes were classified by the phase of the expression peak and organ in which each gene was expressed (phase 2, the peak time $=\mathrm{CT}$ 0.1 4.0; phase 6, CT 4.1 8.0; phase 10, CT 8.1 12; phase 14, CT 12.1 16; phase 18, СТ 16.1 20; phase 22, СТ 20.1 24). The aligned sequences spanning from $1 \mathrm{~kb}$ upstream of the transcription start site (TSS) to the 3' untranslated region in each of human and mouse genes were obtained from the VISTA genome browser (VISTA Browser, VGB2, [41], version: Human, May 2004; Mouse, Aug. 2006), which has pre-computed alignments of whole genome assemblies. Fuzznuc (EMBOSS, [42]), a tool for nucleic acid pattern searching, was used for the first screening; each aligned sequence from VISTA was searched with the following sequence: E-box (CACGTG, $0 \sim 1$ base mismatch) +6 or 7 arbitrary bases + E-box (CACGTG, 0 2 base mismatches). The conserved EE-elements between human and mouse were listed. Subsequently, the EE-elements within $1 \mathrm{~kb}$ upstream region from TSS were listed in Table 2. 


\section{Authors' contributions}

YN and MY carried out experiments and drafted the manuscript. AT discussed the results. MY, HS, and TY carried out the in silico study. AY participated in the coordination and provided financial support. TN carried out the structural design. TT participated in the experimental design and coordination and wrote the paper. All authors read and approved the final manuscript.

\section{Additional material}

\section{Additional file 1}

Effect of the E2 mutant in hPer3 on circadian expression of luciferase. Wild-type (gray letters) and mutant (black letters) sequences of hPer3 EE-element and their flanking sequences are shown in upper panel. The core sequence within the EE-element is shown in capital letters. Bioluminescence monitoring of the hPer3 EE-element-Luc construct was performed by using IV-ROMS (black; mutant, gray; wild-type). The abscissa presents "day"; and the ordinate shows "relative luciferase intensity". First peak values of the curves were set to 1 .

Click here for file

[http://www.biomedcentral.com/content/supplementary/1471-

2199-9-1-S1.pdf]

\section{Additional file 2}

Effect of the EE-element mutant in Per2 promoter on circadian expression of luciferase. Bioluminescence monitoring of the Per2 promoter-Luc construct was performed by IV-ROMS (black; mutant, gray; wild-type). The abscissa presents "day"; and the ordinate indicates "relative luciferase intensity". First peak values of the curves were set to 1. Click here for file

[http://www.biomedcentral.com/content/supplementary/14712199-9-1-S2.tiff]

\section{Additional file 3}

Characterization of BMAL1 antibody. (A) Western blotting. Two bands at around $70-80 \mathrm{kDa}$ that correspond to BMAL1 were observed in mouse liver lysates at 4-hr intervals. CT, circadian time; arrows, BMAL1. (B) Immunoprecipitated and blotted with BMAL1 antibody. WT, liver lysate from wild-type mouse; $K O$, liver lysate from BMAL1-deficeient mouse. CIP, calf intestine alkaline phosphatase; arrows, BMAL1; *, a phosphorylated band.

Click here for file

[http://www.biomedcentral.com/content/supplementary/14712199-9-1-S3.tiff]

\section{Additional file 4}

The EE-element of hDbp promoter is sufficient for circadian rhythm generation. (A) Wild-type and mutant sequence of $\mathrm{hDbp} E 1$ and E2 and their flanking region are shown. The core sequence within the EE-element is shown in capital letters. (B) EE-element-driven luciferase bioluminescence by IV-ROMS. Gray lines indicate bioluminescence of wild type hDbp EE-element; and black lines show those of mutants. The abscissa presents "day"; and the ordinate shows "relative luciferase intensity". First peak values of the curves were set to 1. (C) Wild-type (sp6; gray letters) and mutant (sp4, 5, 7, 8; solid letters) sequences of hDBP EE-element and their flanking sequences are shown. Underlines indicate inserted nucleotides into space of the EE-element. The core sequence within the EEelement is shown in capital letters. (D) EE-element-driven luciferase bioluminescence by IV-ROMS. Black lines indicate bioluminescence of the mutant hDbp EE-elements; and the gray lines show those of wild type. The abscissa presents "day"; and the ordinate shows "relative luciferase intensity". First peak values of the curves were set to 1.

Click here for file

[http://www.biomedcentral.com/content/supplementary/14712199-9-1-S4.pdf]

\section{Additional file 5}

Some protein may bind to E2. Electrophoretic mobility shift assay (EMSA) shows that some protein from cellular lysates binds to E2 (left). The bands from liver lysates in E1m were attenuated with excess of $E 1 \mathrm{~m}$, but not in E2m (right).

Click here for file

[http://www.biomedcentral.com/content/supplementary/14712199-9-1-S5.pdf]

\section{Additional file 6}

The information of constructs and genes used in this study. Upper panel shows the construct sequences used in this study. Middle panel demonstrates the oligonucleotides used in Figure 2C. Bottom panel shows the accession numbers of Pers and Dbp used.

Click here for file

[http://www.biomedcentral.com/content/supplementary/14712199-9-1-S6.pdf]

\section{Acknowledgements}

The authors wish to thank Chiaki Matsubara, Setsuko Tsuboi, Yoko Sakakida, and Fumiyuki Hatanaka for their technical assistance and thank an anonymous reviewer for providing interesting interpretation. We are grateful to Teruya Tamaru and Toshiya Inaba for providing the antibodies against BMALI and E4BP4, respectively and also acknowledge Jacky Lucien Avery for his comments on this manuscript. This work was supported in part by grants from the MEXT, Takeda Science Foundation, Uehara Memorial Foundation, and Nippon Boehringer Ingelheim Co., Ltd.

\section{References}

I. Schibler U, Sassone-Corsi P: A web of circadian pacemakers. Cell 2002, III:919-922

2. Hastings MH, Reddy AB, Maywood ES: A clockwork web: circadian timing in brain and periphery, in health and disease. Nat Rev Neurosci 2003, 4:649-66l.

3. Yamamoto T, Nakahata Y, Soma H, Akashi M, Mamine T, Takumi T: Transcriptional oscillation of canonical clock genes in mouse peripheral tissues. BMC Mol Biol 2004, 5: 18.

4. Schibler U: The daily rhythms of genes, cells and organs. Biological clocks and circadian timing in cells. EMBO Rep 2005, 6 Spec No:S9-I3. 
5. Fu L, Lee CC: The circadian clock: pacemaker and tumour suppressor. Nat Rev Cancer 2003, 3:350-36I.

6. Bell-Pedersen D, Cassone VM, Earnest DJ, Golden SS, Hardin PE, Thomas TL, Zoran MJ: Circadian rhythms from multiple oscillators: lessons from diverse organisms. Nat Rev Genet 2005, 6:544-556.

7. $\mathrm{Ko} \mathrm{CH}$, Takahashi JS: Molecular components of the mammalian circadian clock. Hum Mol Genet 2006, 15 Spec No 2:R27I-7.

8. Wijnen H, Young MW: Interplay of circadian clocks and metabolic rhythms. Annu Rev Genet 2006, 40:409-448.

9. Ueda HR, Hayashi S, Chen W, Sano M, Machida M, Shigeyoshi Y, lino $M$, Hashimoto S: System-level identification of transcriptional circuits underlying mammalian circadian clocks. Nat Genet 2005, 37:187-192.

10. Gekakis N, Staknis D, Nguyen HB, Davis FC, Wilsbacher LD, King DP, Takahashi JS, Weitz CJ: Role of the CLOCK protein in the mammalian circadian mechanism. Science 1998, 280: I564- I569.

II. Kume K, Zylka MJ, Sriram S, Shearman LP, Weaver DR, Jin X, Maywood ES, Hastings MH, Reppert SM: mCRYI and mCRY2 are essential components of the negative limb of the circadian clock feedback loop. Cell 1999, 98:193-205.

12. Hida A, Koike N, Hirose M, Hattori M, Sakaki Y, Tei H: The human and mouse Period I genes: five well-conserved E-boxes additively contribute to the enhancement of mPerl transcription. Genomics 2000, 65:224-233.

13. Jin X, Shearman LP, Weaver DR, Zylka MJ, de Vries GJ, Reppert SM: A molecular mechanism regulating rhythmic output from the suprachiasmatic circadian clock. Cell 1999, 96:57-68.

14. Ripperger JA, Shearman LP, Reppert SM, Schibler U: CLOCK, an essential pacemaker component, controls expression of the circadian transcription factor DBP. Genes Dev 2000 14:679-689.

15. Etchegaray JP, Lee C, Wade PA, Reppert SM: Rhythmic histone acetylation underlies transcription in the mammalian circadian clock. Nature 2003, 421:I77-182.

16. Triqueneaux G, Thenot S, Kakizawa T, Antoch MP, Safi R, Takahashi JS, Delaunay F, Laudet V: The orphan receptor Rev-erbalpha gene is a target of the circadian clock pacemaker. J Mol Endocrinol 2004, 33:585-608.

17. Ripperger JA, Schibler U: Rhythmic CLOCK-BMALI binding to multiple E-box motifs drives circadian Dbp transcription and chromatin transitions. Nat Genet 2006, 38:369-374.

18. Preitner N, Damiola F, Lopez-Molina L, Zakany J, Duboule D, Albrecht $U$, Schibler $U$ : The orphan nuclear receptor REV-ERBalpha controls circadian transcription within the positive limb of the mammalian circadian oscillator. Cell 2002, I I 0:25I-260.

19. Sato TK, Panda S, Miraglia LJ, Reyes TM, Rudic RD, McNamara P, Naik KA, FitzGerald GA, Kay SA, Hogenesch JB: A functional genomics strategy reveals Rora as a component of the mammalian circadian clock. Neuron 2004, 43:527-537.

20. Nakajima Y, Ikeda M, Kimura T, Honma S, Ohmiya Y, Honma K: Bidirectional role of orphan nuclear receptor $R O R a$ alpha in clock gene transcriptions demonstrated by a novel reporter assay system. FEBS Lett 2004, 565:122-126.

21. Akashi M, Takumi T: The orphan nuclear receptor RORalpha regulates circadian transcription of the mammalian coreclock Bmal I. Nat Struct Mol Biol 2005, I 2:44|-448.

22. Albrecht U, Sun ZS, Eichele G, Lee CC: A differential response of two putative mammalian circadian regulators, mperl and mper2, to light. Cell 1997, 9 I:1055-1064.

23. Takumi T, Matsubara C, Shigeyoshi Y, Taguchi K, Yagita K, Maebayashi Y, Sakakida Y, Okumura K, Takashima N, Okamura H: A new mammalian period gene predominantly expressed in the suprachiasmatic nucleus. Genes Cells 1998, 3:167-176.

24. Zheng B, Larkin DW, Albrecht U, Sun ZS, Sage M, Eichele G, Lee CC, Bradley $A$ : The $m$ Per2 gene encodes a functional component of the mammalian circadian clock. Nature 1999, 400:169-173.

25. Nakahata $Y$, Akashi M, Trcka D, Yasuda A, Takumi T: The in vitro real-time oscillation monitoring system identifies potential entrainment factors for circadian clocks. BMC Mol Biol 2006, 7:5.

26. Akashi M, Ichise T, Mamine T, Takumi T: Molecular mechanism of cell-autonomous circadian gene expression of Period2, a crucial regulator of the mammalian circadian clock. Mol Biol Cell 2006, 17:555-565.
27. Yoo SH, Ko CH, Lowrey PL, Buhr ED, Song El, Chang S, Yoo OJ, Yamazaki S, Lee C, Takahashi JS: A noncanonical E-box enhancer drives mouse Period2 circadian oscillations in vivo. Proc Nat Acad Sci U S A 2005, 102:2608-26I3.

28. Panda S, Antoch MP, Miller BH, Su Al, Schook AB, Straume M, Schultz PG, Kay SA, Takahashi JS, Hogenesch JB: Coordinated transcription of key pathways in the mouse by the circadian clock. Cell 2002, 109:307-320.

29. Storch KF, Lipan O, Leykin I, Viswanathan N, Davis FC, Wong WH, Weitz CJ: Extensive and divergent circadian gene expression in liver and heart. Nature 2002, 417:78-83.

30. Ueda HR, Chen W, Adachi A, Wakamatsu H, Hayashi S, Takasugi T, Nagano M, Nakahama K, Suzuki Y, Sugano S, lino M, Shigeyoshi Y, Hashimoto S: A transcription factor response element for gene expression during circadian night. Nature 2002, 418:534-539.

3I. Coulson JM, Fiskerstrand CE, Woll PJ, Quinn JP: E-box motifs within the human vasopressin gene promoter contribute to a major enhancer in small-cell lung cancer. Biochem J 1999, 344 Pt 3:961-970.

32. Kimura H, Weisz A, Ogura T, Hitomi $Y$, Kurashima $Y$, Hashimoto $K$, D'Acquisto F, Makuuchi M, Esumi H: Identification of hypoxiainducible factor $I$ ancillary sequence and its function in vascular endothelial growth factor gene induction by hypoxia and nitric oxide. J Biol Chem 200I, 276:2292-2298.

33. McDonald MJ, Rosbash M, Emery P: Wild-type circadian rhythmicity is dependent on closely spaced $E$ boxes in the Drosophila timeless promoter. Mol Cell Biol 200I, 2 I:I207-1217.

34. Nair SK, Burley SK: X-ray structures of Myc-Max and Mad-Max recognizing DNA. Molecular bases of regulation by protooncogenic transcription factors. Cell 2003, I | 2:193-205.

35. Hogenesch JB, Gu YZ, Jain S, Bradfield CA: The basic-helix-loophelix-PAS orphan MOP3 forms transcriptionally active complexes with circadian and hypoxia factors. Proc Natl Acad Sci U S A 1998, 95:5474-5479.

36. Ohno T, Onishi Y, Ishida N: A novel E4BP4 element drives circadian expression of mPeriod2. Nucleic Acids Res 2007, 35:648-655.

37. Lee C, Etchegaray JP, Cagampang FR, Loudon AS, Reppert SM: Posttranslational mechanisms regulate the mammalian circadian clock. Cell 200I, 107:855-867.

38. Munoz E, Brewer M, Baler R: Circadian Transcription. Thinking outside the E-Box. J Biol Chem 2002, 277:36009-360I7.

39. Tamaru T, Isojima $Y$, van der Horst GT, Takei K, Nagai K, Takamatsu $\mathrm{K}$ : Nucleocytoplasmic shuttling and phosphorylation of BMALI are regulated by circadian clock in cultured fibroblasts. Genes Cells 2003, 8:973-983.

40. Kuribara R, Kinoshita T, Miyajima A, Shinjyo T, Yoshihara T, Inukai T, Ozawa $K$, Look AT, Inaba T: Two distinct interleukin-3-mediated signal pathways, Ras-NFIL3 (E4BP4) and Bcl-xL, regulate the survival of murine pro-B lymphocytes. Mol Cell Biol 1999, 19:2754-2762.

4I. VGB2: [http://pipeline.lbl.gov/cgi-bin/gateway2]

42. EMBOSS: [http://bioweb.pasteur.fr/docs/EMBOSS/fuzznuc.html].
Publish with Biomed Central and every scientist can read your work free of charge

"BioMed Central will be the most significant development for disseminating the results of biomedical research in our lifetime. "

Sir Paul Nurse, Cancer Research UK

Your research papers will be:

- available free of charge to the entire biomedical community

- peer reviewed and published immediately upon acceptance

- cited in PubMed and archived on PubMed Central

- yours - you keep the copyright
BioMedcentral 